\title{
Representaciones Sociales sobre el Derecho a Educación: Las demandas del movimiento estudiantil de Chile a través de la mirada del diario nacional El Mercurio durante el periodo 2011 y 2014
}

Henríquez Ayala, Maryan ${ }^{1}$

Recibido: 9 de abril de 2017 / Aceptado: 16 de octubre de 2017

Resumen. El presente artículo propone el análisis de la construcción de sentido que el medio nacional El Mercurio efectuó sobre las demandas del movimiento estudiantil de 2011 y 2014 en Chile, en torno al derecho a la Educación. Se busca identificar tendencias temáticas que permitan develar las representaciones sociales que los textos informativos promovieron. Para ello se utiliza el software Atlas.ti y la triangulación teórica entre la Mediación Social (Martín Serrano, 2009), el Marco de acción Pública (Subirats, Knoepel, Larrue y Varobe, 2012) y el Análisis Crítico del Discurso (Van Dijk, 1998, 2003). Los resultados obtenidos dan cuenta de un marcado enfoque de servicios y una cobertura limitada a materias que abordan la educación como el ejercicio de un derecho.

Palabras clave: Derecho a la educación; representaciones sociales; medios de comunicación; mediación social; producción de sentido.

[en] Social representations on the right to Education: The demands of the Chilean student movement through the vision of the national newspaper El Mercurio during the years 2011 and 2014

\begin{abstract}
This article proposes the analysis of the construction of meaning that the national newspaper El Mercurio made on the demands of the student movement of 2011 and 2014 in Chile, regarding the right to Education. It seeks to identify thematic trends which allows to unveil the social representations that the informative texts promoted. For this purpose, the Atlas.ti software and the theoretical triangulation between Social Mediation (Martín Serrano, 2009), the Public Action Framework (Subirats, Knoepel, Larrue and Varobe, 2012) and Critical Discourse Analysis (Van Dijk, 1998, 2003), were used. The results show a noticeable approach to services and a limited coverage to issues that address education as a right.
\end{abstract}

Keywords: Right to education; social representations; media; social mediation; production of meaning.

Sumario. 1. Introducción. 2. Análisis Teórico. 3. Corpus de análisis y métodos. 4. Análisis y resultados. 5. Discusión y conclusiones. 6 . Referencias bibliográficas.

\footnotetext{
${ }^{1}$ Cámara de Diputados (Chile).

mhenriquez@congreso.cl
} 
Cómo citar: Henríquez Ayala, M. (2017). "Representaciones Sociales sobre el Derecho a Educación: Las demandas del movimiento estudiantil de Chile a través de la mirada del diario nacional El Mercurio durante el periodo 2011 y 2014”, en Mediaciones Sociales, 16, 193-209

\section{Introducción}

El control social que las instituciones que producen y entregan información ejercen sobre las representaciones sociales, observadas desde la perspectiva mediacional de Manuel Martín Serrano (1997, 2008), forma parte del eje central del presente trabajo. Se propone el análisis de la construcción de sentido que el medio nacional El Mercurio efectuó sobre el discurso de los actores sociales, específicamente del movimiento estudiantil de 2011 y 2014 en Chile, en torno al concepto derecho a la educación. Cabe destacar que la selección del medio responde al protagonismo que este presenta en el debate político nacional.

La crítica introducida por los estudiantes en 2011 vino a cuestionar no solo un modelo educativo con más de 30 años de vigencia, sino que además, incorporó en el debate público demandas vinculadas con el ejercicio de derechos sociales como la educación y la participación efectiva.

De esta manera, los actores sociales develaron una crisis del enfoque de mercado que ha direccionado las políticas públicas en materia de educación (Muller, 2002; Cox, 2005 y Donoso, 2005), situación reconocida incluso por las autoridades de gobierno durante la tramitación de reformas a la educación superior en el último periodo. Esta se configura como una oportunidad para plantear la interrogante sobre qué estamos entendiendo por derecho a la educación y cuáles son las representaciones sociales que hoy definen la interpretación que las instituciones mediadoras ofrecen a la comunidad sobre la materia.

Los estudios del discurso mediático abordados desde una perspectiva sociocognitiva (Van Dijk, 1996,1998, 2003; Wodak y Meyer, 2003; Fairclaugh y Wodak, 1997; Martín Serrano, 2008) orientan el análisis de la cognición social en torno al objeto de estudio, aportando herramientas teórico-metodológicas que permiten observar más allá de las rutinas periodística y la práctica productiva presente en la redacción de textos informativos (Alsina, 1999). Asimismo, criterios editoriales para la selección de hechos noticiosos, la percepción de los periodistas (McCombs, 2006) y la lucha de los actores por formar parte de las fuentes de información que dominarán el debate público (Fontcuberta, 1993) se ponen en interacción con las representaciones sociales (Meyer y Wodak, 2003; van Dijk, 1998,2003; Martín Serrano, 2008, 2009) que transmiten dichos discursos para develar la producción de sentido tras la noción de derecho a la educación en tanto garantía constitucional reclamada por el movimiento estudiantil que llega a la sociedad a través de la mediación efectuada por el discurso periodístico. 


\section{Análisis Teórico}

\subsection{Representaciones y Mediación social en la Prensa}

El papel que juegan los medios de comunicación como constructores de realidad (Berguer y Luckman, 2001) y la influencia del discurso mediático como una pieza importante en la formación de marcos colectivos de percepción (Meyer, 2003), es decir, de representaciones sociales, han marcado una línea de investigación en los estudios sobre la comunicación en las últimas décadas.

$\mathrm{Su}$ influencia y capacidad de persuasión ha sido reconocida por diversos autores, que han centrado sus objetos de estudio en el rol mediador de la prensa y en su participación en la producción y reproducción de sentido. Hablamos de una construcción de realidad, por cuanto el discurso mediático, como el discurso en general, es entendido como una práctica social (Fairclough y Wodak, 1997) que al ser observada desde un plano sociocognitivo (van Dijk, 1998, 2003, 2005; Martín Serrano, 2008, Wodak y Meyer, 2003; Fairclough y Wodak, 1997) ha dado cuenta del impacto que este posee en la manera en que los sujetos interiorizan ciertas visiones de mundo, a partir de la información que otros le proporcionan, ya sea de forma personal o por conductos institucionales.

De esta manera, el proceso de selección de los hechos noticiosos, en el que se define lo que es noticia y lo que no lo es, responde a criterios editoriales del medio de comunicación y a la percepción del propio periodista que construye el relato en función de su imaginario. Al identificar un acontecimiento y dotarlo de un carácter noticioso, se orienta la discusión pública demarcando aquello en lo que la ciudadanía debe poner atención para establecer la agenda pública de los medios informativos (McCombs, 2006; Martín Barbero, 2002).

En este contexto, la construcción de realidad y la producción de sentido se forma a partir tanto las rutinas organizativas de los profesionales de los medios, así como de la práctica productiva detrás de la redacción de un texto informativo (Alsina, 1999). En esta dinámica también juegan un papel importante las fuentes de información en las que se respalda el discurso mediático. Estas son parte esencial de la estructura informativa, por cuanto es a partir de ellas que se otorga validez al relato periodístico y se institucionalizan ciertos discursos.

Lo anterior concuerda con lo planteado por Teun van Djik (2009, p. 26) quien destaca que

"Las personas de poder obtienen una cobertura de rutina por parte de los medios masivos de noticias, con lo cual ese poder se confirma y legitima más aún. Sea una forma de poder de mediación cumple su propia función autónoma en la producción y reproducción de las estructuras sociales de poder"

Ciertas instituciones o grupos poseen un acceso privilegiado a entornos de decisión que son cubiertos por los medios de comunicación. El discurso mediático entrega un espacio importante a aquellos que dominan el poder simbólico y el conocimiento, transformándose en portador de sus discursos. Diversos sectores harán lo necesario para aparecer en los medios y dar a conocer su opiniones 
(Fontcuberta, 1993). Por el contrario, otros quedarán relegados y les será difícil alcanzar dicha cobertura mediática.

Por tanto, el aporte que el discurso mediático hace en la construcción de modelos mentales, representaciones sociales y para el reforzamiento de ciertas ideologías a través de las estructuras de la información que se presentan, se ve potenciado por el alcance que este tipo de discurso posee. La presencia de los medios en el debate público es su principal fuente de poder, principalmente, porque llega a un número importante de personas, un grupo extenso y heterogéneo.

Los estudios sobre comunicación pública aportados por Manuel Martín Serrano (2009) han dado cuenta de aquella consonancia entre la acción social, las organizaciones o instituciones y la información, identificando cómo el individuo o el medio que cumple la labor mediacional sugiere una interpretación socialmente aceptable de la realidad, en armonía con las representaciones o visiones del mundo que construyen el consenso colectivo.

Este consenso social al que se hace referencia anteriormente, sustenta la producción de información destinada a la comunicación pública y al uso social, tema abordado por la teoría social de comunicación y la producción social del autor antes referido. Desde esta perspectiva, las representaciones del mundo que se exteriorizan a través de una narración son interiorizadas como una visión de mundo asumida como propia, a través de un proceso de enculturización (Martín Serrano, 2009).

\subsection{Agenda pública y Movimientos Sociales}

Las representaciones sociales, entendidas como "una determinada interpretación de lo que existe o de lo que acontece en el entorno" (Martín Serrano, 2008, p. 57) contienen modelos ideológicos que se reproducen por la acción de una institución mediadora a través de ciertas formas estabilizadas, entendiendo por éstas estereotipos, normas, hábitos, etc. A través de ellas las instituciones imponen un sistema de control social (Berger y Luckmann, 2001) que influye en la construcción de la esfera pública (Habermas, 1981), red de temas y opiniones, cuyos flujos de comunicación permiten aunar criterios, sintetizar y cohesionar temas que marcarán la discusión público-política (Checha y Cabalin, 2016).

Esta construcción colectiva en la que se vinculan percepciones, representaciones, interés y valores de actores con capacidad de resolución (Subirats, Knoepfel, Larrue y Varone, 2012, p.128) conforman el problema público, de allí radica la riqueza de enfoques como los aportados por el Marco de Acción Colectiva y el enfoque cognitivo en políticas públicas para observar de qué manera se incorporó y posicionó en la agenda pública la demanda estudiantil.

Bajo este marco se comprende al Estado en acción y

“a las políticas públicas como la expresión de marcos cognitivos y normativos que constituyen sistemas de interpretación de la realidad en el seno de las cuales los distintos actores públicos y privados pueden situar sus acciones” (Muller, Surel 1998:47. En Subirats, Et al, 2012, p. 24) 
Entre estos actores encontramos agentes mediadores que ocupan una posición estratégica en los entornos de decisión y que aportan un marco intelectual de interpretación del conflicto social. Estos agentes,

"realizan la construcción referencial de una política, es decir, la creación de las imágenes cognitivas que determinan la percepción del problema por parte de los grupos presentes y la definición de las soluciones apropiadas" (Muller, P. 2002, p.85)

Es conocido el impacto que el movimiento estudiantil de 2011 generó en dicha agenda. Este fue antecedido por las movilizaciones de 2006 lideradas por estudiantes secundarios y se proyectan hasta nuestros días en las discusiones sobre las reformas a la educación las cuales suscitaron estudios desde la comunicación en Chile que se han volcado hasta nuestros días al análisis sistémico de su participación como actores que cuestionaron un modelo educativo y reclamaron un espacio en los entornos de decisión. (Gascón i Martín y Pacheco, 2012; Dittus, 2009; González, Cornejo, Caldichoury y Sánchez, 2006; Bellei, Cabalin y Orellana, 2014).

Lo anterior, da cuenta de un objetivo cumplido por parte de los actores sociales, por lo menos en cuanto a la visivilización de una demanda y su transformación a un problema público. Las manifestaciones impulsaron una respuesta por parte de las autoridades (Becker, 1995en Olavarría, 2007), que se concretó no solo en el reconocimiento de la problemática, sino también en cambios a nivel de política pública siendo debatido en el espacio político -administrativo (Gusfield, 1981, 2004). Se enviaron mensajes presidenciales que tomaron la coyuntura como un complemento argumentativo para la instalación de cambios en la legislación en educación, como ocurrió con normas como la Ley General de Educación de 2007, la Ley de Aseguramiento de la Calidad de 2011 y la Ley de inclusión publicada en 2015 .

De esta manera, el movimiento estudiantil originó el cumplimiento de los 4 elementos fundamentales planteados por Subirats et al (2012) para la inclusión de un tema en la agenda pública:

a. Intensidad del problema: Los estudiantes dieron cuenta de un sistema desigual que presenta efectos negativos a nivel individual como colectivo, además de instalar la perspectiva de derechos en el debate al reclamar el ejercicio del derecho a la educación por parte de todos los ciudadanos.

b. Perímetro (o la audiencia) del Problema: Los actores sociales dieron cuenta de un problema en la calidad de la educación que afecta de manera transversal a los distintos estratos sociales, además de procedimientos discriminatorios al interior del sistema, como lo es la selección de los alumnos.

c. La novedad del Problema: Captaron el interés de los medios de comunicación a pesar de que la demanda estudiantil venía gestándose desde inicios del 2000, sin embargo, las estrategias de movilización y la 
instalación de conceptos claves en el discurso por parte de los dirigentes los posicionó en la agenda.

d. Urgencia del problema: Irregularidades en la acreditación de universidades privadas como la Universidad del Mar en 2010 que requirió de la intervención del Estado, se transformó en una ventana de oportunidad para cuestionar la calidad de la educación y visibilizar las demandas.

Si observamos las características del movimiento estudiantil chileno nombradas anteriormente, es posible identificar una clara tendencia a la definición de estos actores como nuevos movimientos sociales (Laraña y Gousffield, 2001), puesto que se preocupan por la calidad, la equidad y la participación. Apuntan hacia necesidades que van más allá de la cobertura educativa o el acceso, entendiéndolo como una mejora en la calidad de vida de las personas y como un derecho universal.

Cabe destacar, que estas demandas surgen en un momento en que el país se encuentra agitado producto de una serie de manifestaciones en oposición a proyectos que afectan el medio ambiente como la instalación de hidroeléctricas en distintos partes del territorio, además del descontento que provocó la lentitud con que se estaba llevando a cabo la reconstrucción tras el terremoto vivido en febrero de 2011, la que a su vez dejó a la vista las precaria infraestructura de un número importante de establecimientos educacionales. Esto también fue acompañado por un escenario internacional de movilizaciones liderado por el $15 \mathrm{M}$ en España, con una demanda por mayor representatividad y participación.

Este despertar social (Garcés, 2012) evidenció, además, los escasos espacios institucionales de participación permanente que integran a los actores sociales en los entornos de decisión. Por lo general, estos suelen responder a una coyuntura específica, situación que da cuenta del escaso protagonismo de los ciudadanos en el diseño, ejecución y evaluación de políticas públicas (Garretón, 2010).

\section{Corpus de análisis y métodos}

Para el análisis de las representaciones sociales sobre el derecho a la educación presentes en las demandas del movimiento estudiantil que fueron publicadas por el diario nacional El Mercurio, se revisaron los textos informativos pertenecientes a las secciones nacional y reportajes, debido a la relevancia que ambas otorgan al tratamiento de fuentes de información y a las características propias del genero informativo.

El periodo estudiado, comprendió los meses de marzo a diciembre del año 2011, mientras que en 2014 se revisaron los meses de marzo a noviembre, en consideración al espacio de tiempo en que los actores sociales obtuvieron mayor visibilidad al interior de las publicaciones.

La selección del corpus de investigación se realizó en dos etapas: En una primera instancia se seleccionaron aquellas informaciones que hacían mención al movimiento estudiantil, las que alcanzaron un total de 530 noticias para 2011 y 391 en 2014. En una segunda etapa, estas fueron clasificadas en tres categorías: 
a. Incorporan como fuente a estudiantes: Registró 217 textos informativos en 2011 y 106 en 2014.

b. No incorporan a estudiantes como fuente: Alcanzó las 245 publicaciones en 2011 y 65 en 2014

c. Referidas únicamente a la reforma educacional: totalizaron 68 textos informativos en 2011 y 220 en 2014.

Dado que el fin de analizar los textos informativos fue conocer el modo en que la prensa construyó públicamente el discurso de los estudiantes, se seleccionó para el estudio la categoría "Incorporan como fuentes a estudiantes", con un total de 323 textos informativos. De ellos, se consideraron para el análisis aquellas que hacen referencia directa o indirecta a las demandas de los estudiantes, quedando el corpus compuesto por un total de 130 piezas, de las cuales 84 son de 2011 y 46 de 2014 .

Las publicaciones fueron codificadas y sistematizadas a través del Software Atlas.ti, con el fin de identificar los términos que se asociaban con mayor regularidad a los conceptos Derecho Educación y Equidad. De esta manera, el procesamiento de datos se efectuó en cuatro fases (Nicolini, 2015): En una primera aproximación se llevó a cabo un pre análisis que situó de manera genérica los conceptos en cuestión. Posteriormente, en una segunda fase, se llevó a cabo una codificación abierta (San Martin, 2014) utilizando el criterio de aparición de los conceptos en estudio, en función de las menciones explícitas e indirectas.

Posteriormente, se categorizaron los resultados permitiendo identificar tendencias temáticas que guiaron una nueva codificación conceptual, lo que arrojó un listado de códigos que vinculan a la categoría principal una serie de temas considerados como subcategorías.

\section{Subcategorías Derecho a la Educación}

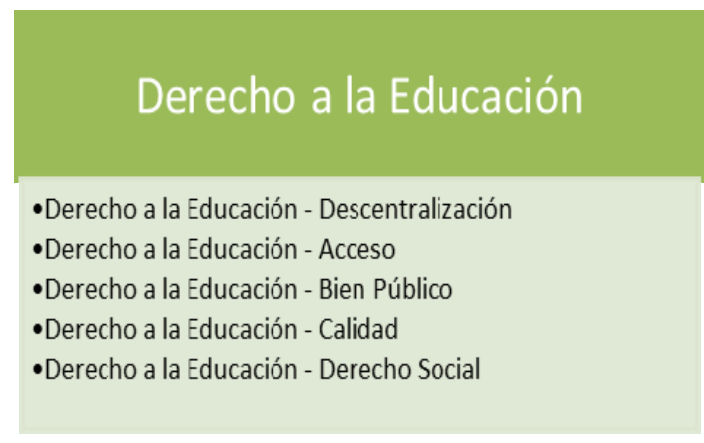

Fuente: elaboración propia

Aplicada la codificación y categorización se procesaron los datos, siendo posible cuantificar el número total de apariciones de cada categoría, identificar aquellas que concentraron el mayor número de menciones, para finalmente, realizar un análisis cualitativo de la información obtenida, a través de la herramienta de 
Redes o Network, la que permitió visualizar esquemas de relaciones de las citas contenidas en los códigos, otorgando la posibilidad de crear redes semánticas.

En función del esquema antes descrito fue posible aproximarnos no solo a los significados atribuidos a las dimensiones derecho a la educación y equidad, sino que además, adentrarnos a la construcción discursiva que el diario nacional El Mercurio realizó durante ambos años en lo referido a la selección de los hechos noticiosos y su consideración de las demandas estudiantiles.

\section{Análisis y Resultados}

La codificación de los textos informativos que componen el corpus de análisis evidencia una fuerte presencia de la Categoría Derecho a la Educación en las demandas estudiantiles publicadas por el diario nacional El Mercurio, durante el 2011 y el 2014. Se registraron un total de 345 referencias lo que da cuenta de la cobertura entregada por el diario analizado.

Con el fin de responder al objetivo propuesto, tras identificar la presencia de ambas categorías en los textos informativos, se prosiguió a codificar subcategorías en función de las siguientes tendencias temáticas:

- Derecho a la Educación: Descentralización - Acceso - Bien Público - Calidad - Derecho Social

Al observar de forma particular los resultados obtenidos de las subcategorías relacionadas con el Derecho a la Educación, es posible notar una clara tendencia al abordaje de materias que vinculan a esta garantía con el bien público. Entre ellas se encuentran las que hacen referencia al aporte de la educación al país, aquellas que se relacionan con los recursos asociados y con la provisión de la educación.

\section{Referencias contenidas en los textos informativos según año, expresado en porcentajes (\%)}

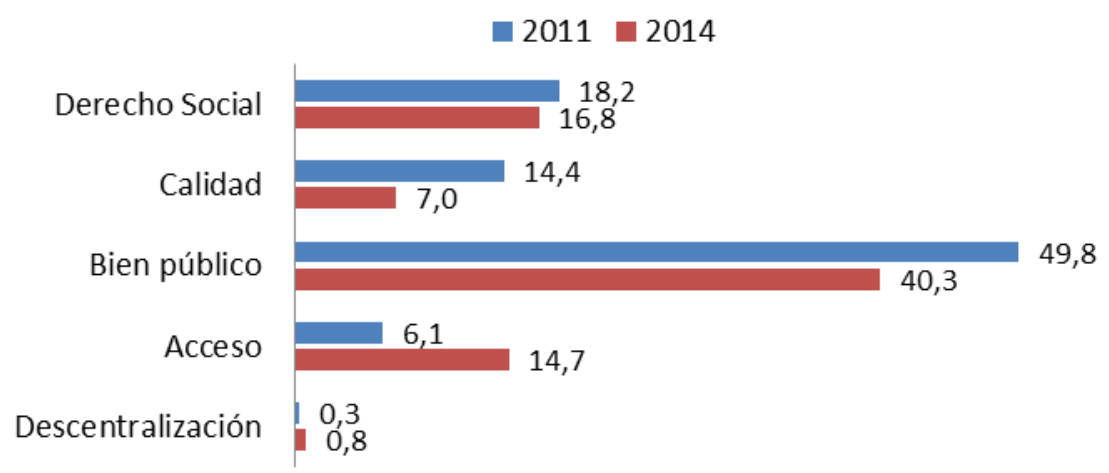

Fuente: Elaboración propia con resultados obtenidos de la codificación en Atlas.Ti 
Así, el Bien Público registró un total de 208 menciones, de las cuales 156 corresponden al año 2011. Muy por debajo, le sigue el Derecho Social con un total de 77 referencias, de las cuales 57 pertenecen a informaciones de 2011, mientras que solo 20 son de 2014 .

En tercer lugar se observan resultados disímiles en función del año analizado. Por una parte, en el año 2011 las menciones sobre calidad registraron una mayor presencia, mientras que en 2014 este tópico pierde fuerza y se posicionan en el debate las referencias asociadas al acceso a la educación.

La frecuencia de apariciones de las subcategorías registradas, entregan luces sobre una concentración de las demandas publicadas por el medio de comunicación en materias relacionadas con los recursos destinados a la educación, ya sea en función de los sistemas de financiamiento, las críticas al lucro, la provisión del derecho o la infraestructura, todas las cuales apuntan a herramientas para garantizar un servicio.

Como una manera de presentar con mayor claridad las tendencias temáticas contenidas en las subcategoría con mayor presencia en las publicaciones, el Bien Público, se presenta a continuación el siguiente esquema:

\section{Tendencias temáticas sobre Bien Público en los textos analizados}

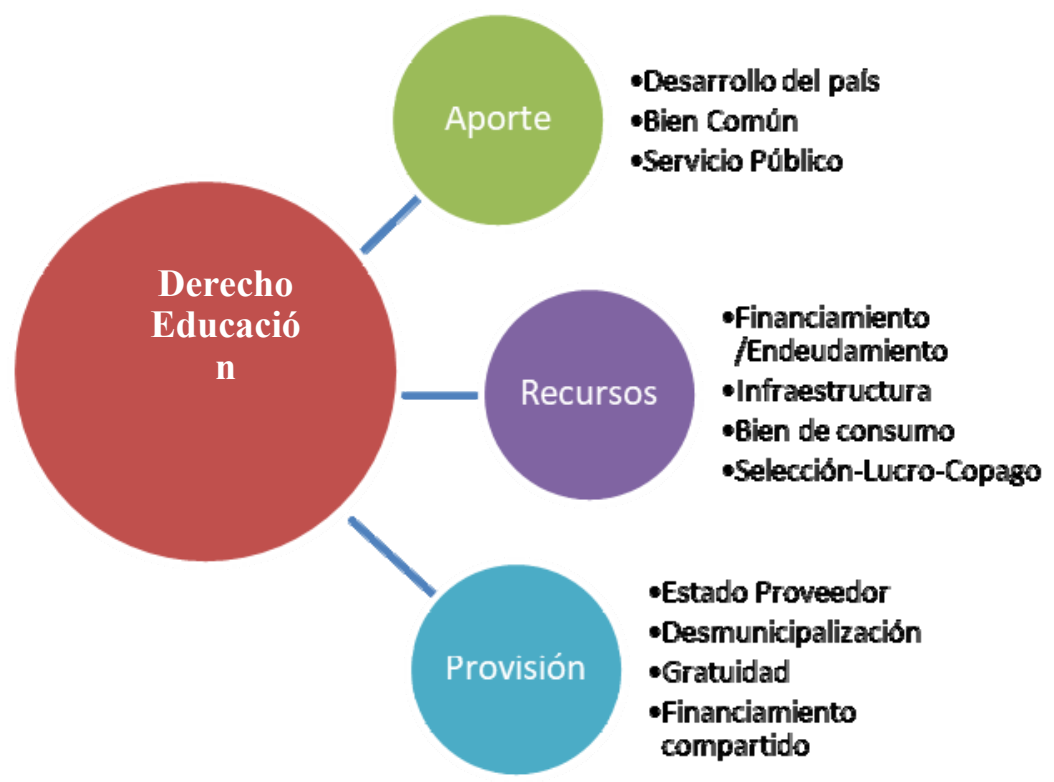

Fuente: Elaboración propia con resultados obtenidos de la codificación en Atlas.Ti

Al realizar una revisión pormenorizada de los contenidos de esta subcategoría, es posible destacar que un número importante de las citas a estudiantes incorporadas en los textos informativos se centran en el financiamiento de la educación. En ellos es recurrente encontrar alusiones al sistema de becas y créditos, 
al endeudamiento de los estudiantes y sus familias y las desigualdades que esto ha generado.

"Sé que tengo que pagar, lo tengo más que claro. Pero no se me ocurre en qué momento mi educación se transformó en un gran negocio" (17.06.2011).

"Afortunadamente pueden costearme la carrera, pero esa no es la realidad de todos mis compañeros ya que muchos se sacrifican para costear sus estudios", agregó” (17a.06.2011).

Como es posible observar en los extractos destacados, el discurso mediático pone énfasis en la responsabilidad que le cabe a padres o estudiantes en el financiamiento de sus estudios, dando cuenta de un problema individual que afecta a una parte de la población. De esta manera, se apunta hacia un sistema educativo con un enfoque privado, en el que la participación del Estado se restringe a un apoyo a los sectores más vulnerables, naturalizando las divisiones al interior de éste en función de los recursos de cada familia.

Como segundo tópico con mayor presencia destaca entre las demandas de los estudiantes las centradas en la provisión, específicamente sobre el rol del Estado como proveedor, en las que se ya se reúnen las menciones sobre gratuidad, desmunicipalización de los colegios para que vuelvan a ser de administración directa del Estado. Asimismo, se integran los cuestionamientos al financiamiento compartido en el que los padres aportan dinero adicional a la subvención que el Estado entrega a los sostenedores privados para financiar la matrícula de un alumno con menos recursos.

Las demandas centradas en la provisión introdujeron al debate la concepción de educación como bien público en el que como tal, el Estado es responsable de garantizar el acceso a toda la población. Bajo esta premisa, persisten dos enfoques, aquel que exige una mayor inversión focalizada en las instituciones públicas y otro que establece una educación gratuita universal a la que se accede en tanto ciudadano.

En tercer lugar, las referencias se concentran en la demanda por el fin al lucro que se ha clasificado bajo el ítem Recursos. Este fue uno de los sellos del movimiento estudiantil de 2011, que promovió entre sus consignas una materia que no había sido integrada por los actores sociales anteriores. Esta venía a reforzar la crisis del sistema, dando cuenta de un tratamiento de la educación como un negocio, un área productiva más desde la cual se obtenían ganancias y utilidades que no estaban siendo reinvertidas en mejorar la calidad de la formación impartida, la que se mantendría vigente en el petitorio de las manifestaciones de 2014.

"La joven explicó que una de las acciones que se estudia es que de las mesas de trabajo surja un documento en común, que tendrá ya como uno de los ejes la demanda de fin al lucro "efectivo en todos los niveles de la educación". Así, los dirigentes buscarán que se prohíban las ganancias en los centros de formación técnica e institutos profesionales, además de los colegios. En esa línea, no comparten el programa de Gobierno de acabar con el lucro solo donde hay fondos públicos” (18.03.2014). 
Muy por debajo de los tópicos antes señalados, se encuentran las referencias al bien público entendido como un aporte al desarrollo del país, en el que la educación se presenta como un elemento esencial para la vida en sociedad y que como tal es de principal relevancia para el bien común. Esta posición suele ser utilizada como un refuerzo de materias como la gratuidad en tanto se considera que la formación es una tarea que debe ser comprendida como un servicio público.

\begin{abstract}
"De esta manera, los cuestionamientos al funcionamiento del sistema educativo, en términos de la segregación que éste ha generado, ha tomado especial relevancia en función del crecimiento descontrolado de la oferta educativa y han marcado el debate de los últimos años sobre esta materia. No obstante, no existe consenso, ni se suelen hacer referencias explícitas a terminar con la participación de privados en la provisión de educación. Se puede observar demandas y acuerdos entorno a la regulación de su funcionamiento, no así sobre la abolición de su participación”. (Henríquez, 2017.p.167)
\end{abstract}

\title{
4.1 La concepción de derecho en educación
}

Otra de las tendencias temáticas con mayor presencia en el debate fue la incorporación de la noción de derechos, en el que se agruparon bajo la subcategoría Derecho Social 77 referencias entre 2011 y 2014, las que representan el 18,2\% de las referencias obtenidas para el primer año y el $16,2 \%$ para el segundo.

Si bien se posiciona muy por debajo de la subcategoría Bien Público, integra al debate una mirada centrada en la educación como una garantía reconocida en la constitución. En ella, materias como la universalidad, la participación y la igualdad agrupan inquietudes de los estudiantes que avanzan a la concepción de un sujeto de derechos activo, interesado en ser un aporte al desarrollo del país a través del ejercicio de su ciudadanía y que como tal reclama espacios para todos sin discriminación. A continuación se presenta un esquema temático que le sintetiza: 


\section{Tendencias temáticas sobre Derecho Social en los textos analizados}

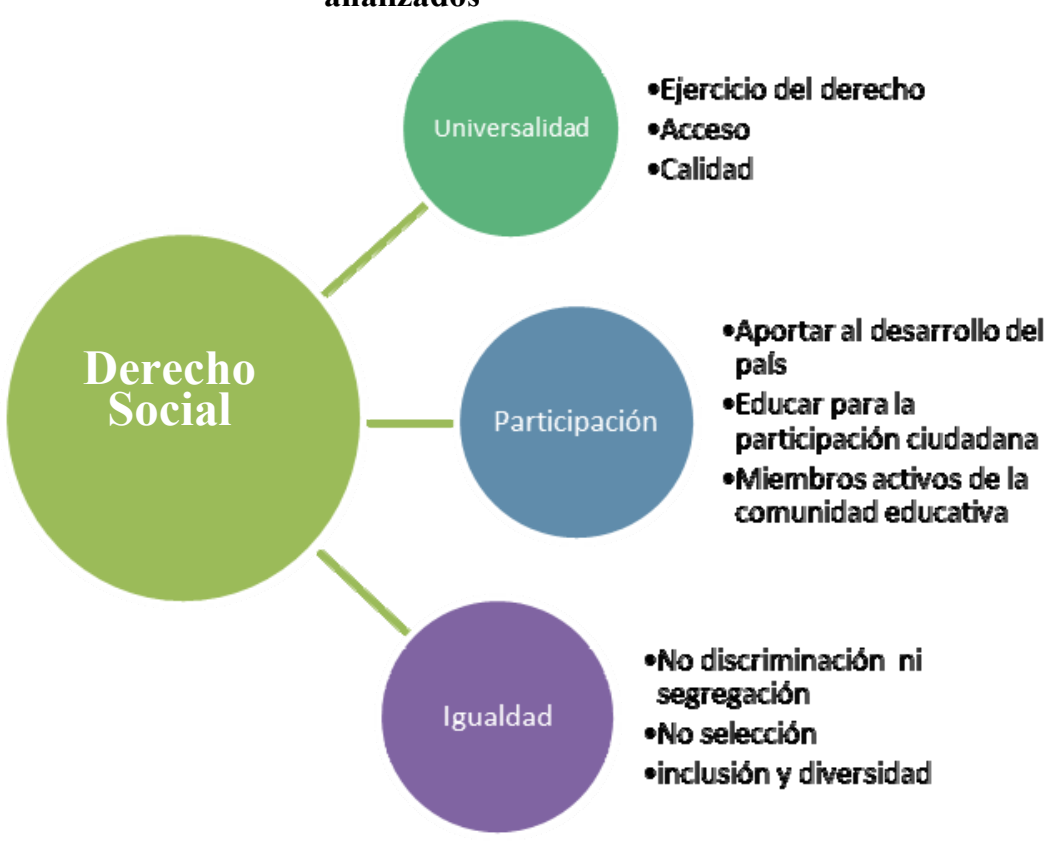

Fuente: Elaboración propia con resultados obtenidos de la codificación en Atlas.Ti

Si bien el concepto Derecho Social es nombrado como tal solo en cuatro oportunidades, una en 2011 y 3 en 2014, esta suele asociarse al aporte que la educación entrega al desarrollo del país.

“1.- La Educación garantizada constitucionalmente como Derecho Social, entendiéndola como plataforma de construcción del conocimiento al servicio del desarrollo social, cultural y económico de nuestro país, donde se apunte a una Educación pública gratuita y de calidad al servicio del país" (30.08.2011).

Entre los tópicos integrados en esta subcategoría, el que obtuvo mayor presencia fue el que hace mención al ejercicio del derecho a educación, consagrado en la Constitución de la República y en el tratado de Derechos Sociales, Económicos, y Culturales, a los que Chile se encuentra suscrito desde 1989. Observado desde la noción de universalidad, este es exigido por los actores sociales en tanto ciudadanos, siendo utilizado habitualmente para poner en cuestión la exclusión y desigualdad que el sistema vigente generaba.

Por otra parte, la demanda por mayor democratización de los gobiernos estudiantiles se posiciona como el segundo tópico con mayor frecuencia en el discurso estudiantil publicado por el diario El Mercurio. En función del ejercicio ciudadano, se reclama la incorporación activa de los estudiantes y de la comunidad 
educativa en general, en la toma de decisiones de los establecimientos educacionales en sus distintos niveles de enseñanza.

Cabe destacar, que la demanda por participación, si bien surge en 2011, será durante el año 2014, donde cobrará mayor relevancia en la cobertura mediática, a través de relatos como el siguiente:

"Otro de los puntos donde hay diferencias es en la denominada democratización o participación de las comunidades en el gobierno de los planteles -uno de los puntos centrales del petitorio de la Confech-, ya que no está considerado en el programa de gobierno y que, si bien es relevante para el Ejecutivo, no hay una propuesta específica”

Por otra parte, la Calidad también estuvo presente en el petitorio de los estudiantes, siendo la tercera subcategoría más abordada por el medio en 2011, al reunir el $14,4 \%$ de las referencias. Tras el análisis, fue posible evidenciar una marcada tendencia a incorporar el concepto en declaraciones generales como "Educación gratuita y de Calidad", sin entregar mayores detalles sobre qué se entiende por ella.

Sin embargo, al revisar las materias asociadas a su uso, fue recurrente encontrar vínculos con demandas por mejoras en infraestructura y equipamiento para los establecimientos educacionales. Lo anterior, podría deberse en parte, a las consecuencias del terremoto que afectó al país en febrero de 2010, evento que dejó a la vista las precarias construcciones de un número importante de establecimientos educacionales.

Para el año 2014, la subcategoría que se posicionó en el tercer lugar, según el porcentaje de referencias obtenidas de la codificación fue el concepto de Acceso, el que alcanzó el $14,7 \%$. Durante este periodo, a las demandas por gratuidad en educación superior de 2011, se incorporan temas como la eliminación de los procesos de selección de estudiantes para el ingreso a establecimientos de educación primaria y secundaria, además de las críticas a la Prueba de Selección Universitaria (PSU).

\section{Discusión y Conclusiones}

Desde un plano general, la voz de los actores sociales encontró un espacio en las publicaciones, en función de una coyuntura marcada por las manifestaciones que se abrieron un lugar en el debate público durante el primer periodo estudiado. Así, el diario nacional El Mercurio integró como fuente a estudiantes en el $40 \%$ de los textos informativos que abordaron el movimiento estudiantil en 2011, mientras que en 2014, esta cifra solo alcanzó el $27 \%$.

En cuanto al enfoque entregado por el medio, la evidencia indica que este se concentró en aquellas materias del petitorio asociadas al financiamiento de la educación, ya sea en el acceso a crédito o becas, en el endeudamiento de las familias, en la infraestructura y el lucro en la educación.

Todo lo anterior, enfoca las demandas estudiantiles principalmente en los recursos necesarios para alcanzar el derecho a la educación, construyendo el relato de los estudiantes a partir de una postura instrumental, que refuerza una noción de 
servicios educacionales. De esta manera, el discurso mediático -a través de la cobertura entregada- participa en la producción de sentido de un dilema utilitario de la demanda social (Riveros, Orellana y Salazar,2014) que refuerza el modelo de mercado educativo y restringe las voces que observan a la educación como un derecho.

El Mercurio -como mediador social- promovió representaciones sociales que sitúan a la educación como un problema individual, donde las familias en función de los recursos económicos que disponen, demandan mayor apoyo por parte del Estado para entregar educación. A través de su discurso, naturaliza una visión neoliberal que pone a la competencia como el principal regulador del mercado. En este esquema, las personas vulnerables deben recibir una ayuda adicional, legitimando con ello la desigualdad social que el sistema educativo genera y limitando al Estado a un papel de facilitador del acceso a enseñanza por medio de una subvención, de becas o créditos.

Las referencias sobre la calidad de la educación presente en los textos informativos, también dieron cuenta del fomento de una noción de servicios, en tanto relacionaban el concepto a mejoras en la infraestructura y equipamiento tecnológico de los establecimientos educacionales, alineada con la visión utilitarista mencionada anteriormente.

Se construye, por tanto, una perspectiva del movimiento estudiantil que le posiciona entre los límites impuestos por el enfoque neoliberal. Se invisibiliza el enfrentamiento ideológico entre los actores sociales que exigen transformaciones profundas al sistema y las autoridades de gobierno (Henríquez, 2017).

Así, el discurso mediático construye un cierto consenso y legitima un modelo que se proyecta a la opinión pública, desde una posición privilegiada (Van Dijk, 2009), cuyo poder radica en la posición valorada que la sociedad le atribuye al diario y en la dominación sobre la construcción de significado que se ejerce sobre los sentidos por él propuesto (García, en Szurmuk y Mckee, 2009).

El poder simbólico (Bourdieu, 2000) del medio de comunicación, se ejerce a través del control social que practica sobre las representaciones sociales por él promovidas, vale decir, sobre las interpretaciones que entrega sobre lo que acontece (Martín Serrano, 2008).

De este modo, la escasa presencia de demandas asociadas a la subcategoría derecho social en los textos informativos, que alcanzó entre el 16\% y el 14\% de las publicaciones en 2011 y 2014, respectivamente, entrega luces sobre una cobertura limitada a materias que abordan la educación como el ejercicio de un derecho -y que como tal- critican la visión de mercado educativo.

La posición ideológica del medio, conservador en lo valórico y neoliberal en lo económico, se alinea con el exiguo especio entregado a la publicación de demandas que exigen un abordaje integral de la educación, que -en función del aporte que esta genera para el desarrollo del país- entregue formación en valores democráticos como la participación, la igualdad y la no discriminación.

El petitorio sobre mayor democracia al interior de los gobiernos educativo en los distintos niveles de enseñanza, junto a la participación de los actores sociales involucrados en el proceso formativo en los entornos de decisión sobre reformas educativas, avanzan hacia un giro en la concepción del derecho a la educación como un derecho social. 
Este exige un compromiso por parte del Estado, como garante del bien común, en la entrega de oportunidades educativas para todas las personas, como parte del ejercicio efectivo de un derecho ciudadano. Esta visión avanza en una línea similar a la promovida por el Pacto Internacional de Derechos Económicos, Sociales y Culturales, que aborda aquellos derechos humanos que garantizan las condiciones básicas para una vida digna y libre, ratificado por Chile en 1989 y cuyo enfoque se ha ido integrando progresivamente en los discursos sobre educación realizados por la Presidenta de la República durante los últimos años en función de las demandas de los actores sociales.

\section{Referencias Bibliográficas}

Alsina, M. R. (1999). La construcción de la noticia. Barcelona, España: Editorial Paidós.

Berger, P. y Luckmann, T. (1999). La construcción social de la realidad. Editorial Amorrortu, Buenos Aires.

Bellei, C., Cabalín, C., Orellana, V. (2014). The 2011 Chilean student movement against neoliberal educational policies. Publicado en Studies in Higher Education. Vol.39, $\mathrm{N}^{\circ} 3$, Pp.426-440. Recuperado de: http://movimientoestudiantil.cl/wpcontent/uploads/2015/12/Bellei-Cabalin-Orellana-Chilean-student-movement-SHE2014.pdf

Bourdieu, P. (2000). Poder, derecho y clases sociales. Palimpsesto. Ed. Desclée de Brouwer.

Cabalin, C. y Antezana, L. (2016). La educación en portada: la visualización de la política educacional en la prensa. Cuadernos.info, Nº39, Pp. 195-207.

Checa, L. y Cabalin, C. (2016). La construcción de la esfera pública política: Un estudio de los cuerpos de reportajes e la prensa chilena. En Eje Temático: Estudios de Periodismo e Historia de Medio, en 3er. Congreso INCOM Chile, Asociación chilena de investigadores en comunicación. Creatividad e innovación para investigar la comunicación. 3 y 4 de noviembre de 2016. Concepción.

Cox, C. (2005). Políticas educacionales en el cambio de siglo. La reforma del sistema escolar en Chile. Santiago, Chile: Universitaria.

De Fontcuberta, M. (1993). La noticia. Pistas para percibir el mundo. Ediciones Paidós Ibérica. Barcelona, España.

Dittus, R. (2009) La Revolución de los Pingüinos en la tesis del contrato de lectura. Jornadas Binacionales de Semiótica. Museo de Arte Contemporáneo. Recuperado en: https://goo.gl/BiRA2q

Donoso Díaz, S. (2005). Reforma y política educacional en Chile 1990-2004: El neoliberalismo en crisis. Estudios Pedagógicos Vol. 31, N¹. Pp. 113-135.

Fairclough, N. y Wodak, R. (1997) Análisis crítico del discurso. En Van Dijk, T. (Comp). (2008). Discurso como interacción social. Estudios sobre el discurso II: una introducción multidisciplinaria. Barcelona, España: Gedisa.

Fontcuberta, M. (1993). La Noticia, Pistas Para Percibir el Mundo. Barcelona, España: Ediciones Paidós. 
Garcés, M. (2012). El despertar de la Sociedad: Los movimientos sociales en América Latina y Chile. Santiago, Chile: LOM Ediciones.

Garretón, M. A. y Garretón, R. (2010). Democracia Incompleta en Chile: la realidad tras los rankings internacionales. Revista de ciencia política / Instituto de Ciencia Política, Universidad Católica de Chile. (Santiago, Chile).Vol. 30, no.1, p. 115-148.

Garretón, M. A., Cruz, M. A., AguirrE, F., Bro, N., Farías, E., Ferreti, P. y Ramos, T. (2011). Movimiento social, nuevas formas de hacer política y enclaves autoritarios: Los debates del Consejo Asesor para la Educación en el gobierno de Michelle Bachelet en Chile. Polis (Santiago), 10(30), 117-140. https://dx.doi.org/10.4067/S071865682011000300006

Gascón, F. y Pacheco, C. (2012). Construcción discursiva de las actorías en las crónicas informativas sobre el movimiento estudiantil en El Mercurio de Valparaíso y El Martutino durante los meses de mayo a diciembre de 2011. COMUNICACIÓN Y MEDIOS N ${ }^{\circ} 25$ (2012). Instituto de la Comunicación e Imagen. Universidad de Chile. Pp. 115-129.

González, J., Cornejo, R. Sánchez, R. y Caldichoury, J. P. (2006). Perspectivas y significados del movimiento nacional de estudiantes secundarios chilenos. Observatorio Chileno de Políticas Educativas, Universidad de Chile. Recuperado en:

http://www.opech.cl/bibliografico/doc_movest/significados $\% 20 \mathrm{del} \% 20$ movimiento $\% 20$ nacional\%20de\%20estudiantes\%20secundarios\%20-\%20OPECH.pdf

Gusfield, J. (2004). La cultura de los problemas públicos. El mito del conductor alcoholizado versus la sociedad inocente. Siglo XXI Editores.

Habermas, J. (1981). Historia y crítica de la opinión pública: la transformación de la vida pública. Barcelona; Santiago de Chile: Gustavo Gili.

Henríquez, M. (2017). Representaciones sobre el concepto de derecho a la educación y equidad en Chile: análisis crítico de los discursos legislativo de las Leyes $\mathrm{N}^{\circ} 20.370$, 20.529 y 20.845 y en los textos informativos del diario El Mercurio de 2011 y 2014 (Tesis Doctoral). Universidad complutense de Madrid, España.

Henríquez, M. (2011). Clic Activismo: redes virtuales, movimientos sociales y participación política. F@ro, Revista Teórica de la Facultad de Ciencias Sociales Universidad de Playa Ancha. Monográfico Año 7, N 13, I semestre. Pp. 29-41.

Laraña, E. y Gusfield, J. (2001). Los nuevos movimientos sociales. De la ideología a la identidad. Madrid, España. Centro de Investigaciones Sociológicas (CIS).

Londoño, O. (2009). El poder del discurso y el discurso del poder. Entrevista a Teun A. van Dijk. Revista Onomázein 20 (2009/2): 195-210.

Lippmann, W. (2003). La opinión pública. Editorial Cuadernos de Langre. Madrid, España.

Martín Barbero, J. (2002). El Oficio de Cartógrafo: Travesías Latinoamericanas de la Comunicación en la Cultura. Santiago, Chile. Fondo de Cultura Económica.

Henríquez, M. (2008). La mediación Social. Edición conmemorativa del 30 aniversario. Ed. Akal, Madrid.

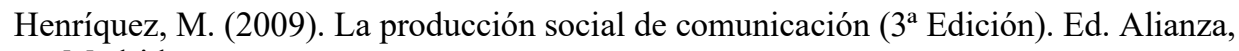
Madrid.

McCombs, M. (2006) Estableciendo la Agenda: El Impacto de los Medios en la Opinión Pública y en el Conocimiento. Paidós. Barcelona. 
Meyer, M. y Wodak, R. (2003). Métodos de análisis crítico del discurso. Barcelona, España: Gedisa.

Muller, P. (2002). Las políticas públicas. Colombia: Universidad Externado de Colombia.

Olavarría, M. (2007). Conceptos básicos en el análisis de políticas públicas. Documentos de Trabajo $\mathrm{N}^{\circ} 11$. Diciembre de 2007. Instituto de asuntos públicos. Departamento de Gobierno y gestión Pública. Universidad de Chile

Parsons, W. (2007). Políticas Públicas. Una introducción a la teoría y la práctica del análisis de políticas públicas. México D.F.: Editorial FLACSO.

Riveros, L., Orellana, J. C. y Salazar, M. (2014). La educación chilena. El dilema de un cambio necesario. Santiago, Chile. Editorial Occidente

Rodrigo Alsina, M. (1990): La construcción de la noticia. Barcelona, España. Paidós.

Richard, N. y Ruiz, C. (2000). Políticas y Estéticas de la Memoria. Santiago, Chile. Cuarto Propio.

Ruiz, C. (2012). La república, el Estado y el mercado en educación. Revista de filosofía, 68, 11-28. https://dx.doi.org/10.4067/S0718-43602012000100003

Subirats, J., Knoepfel, P., Larrue, C. y Varone, F. (2012). Análisis y gestión de políticas públicas. Barcelona, España: Ariel.

Szurmuk, M. y Mckee, R. (2009). Diccionario de Estudios Culturales Latinoamericanos. México D.F. Siglo XXI Editores.

Van Dijk, T. (1998). Texto y contexto. Madrid, España. Editorial Cátedra.

Van Dijk, T. (1996). Análisis del discurso Ideológico. Revista Versión, Estudios de Comunicación y Política. N6, Universidad Autónoma Metropolitana, México. Pp. 1543.

Van Dijk, T. (Comp). (2000). El discurso como estructura y proceso. Estudios sobre el discurso I, una introducción multidisciplinaria. Barcelona: Editorial Gedisa.

Van Dijk, T. (2003). Ideología y discurso. Barcelona, España: Editorial Ariel Lingüística.

Van Dijk, T. (2003). La multidisciplinariedad del análisis crítico del discurso: un alegato a favor de la diversidad. Wodak, Ruth Y Meyer, Michael. Métodos de análisis crítico del discurso. Barcelona, España: Editorial Gedisa.

Van Dijk, T. (2005) Discurso, conocimiento e ideología. CIC (Cuadernos de Información y Comunicación). $\mathrm{N}^{\circ} 10$. Pp.285-318.

Van Dijk, T. (2008). Semántica del discurso e Ideología. Discurso \& Sociedad. Vol. 2 (1), pp.201-261.

Van Dijk, T. (2009). Discurso y Poder. Contribuciones a los Estudios Críticos del Discurso. Barcelona, España: Editorial Gedisa.

Wodak, R. y Meyer, M. Métodos de análisis crítico del discurso. Barcelona, España: Editorial Gedisa. 